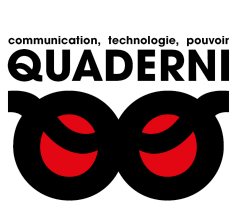

\title{
Quaderni
}

Communication, technologies, pouvoir

\section{En un combat douteux.}

Militantisme en ligne, « complotisme » et disqualification médiatique : le cas de l'association ReOpen911

In Dubious Battle. Online activism, " conspiracism " and disqualification in the media: the French ReOpen911 organization case

Pierre France et Alessio Motta

\section{(2) OpenEdition}

\section{Journals}

Édition électronique

URL : https://journals.openedition.org/quaderni/1103

DOI : 10.4000/quaderni.1103

ISSN : 2105-2956

Éditeur

Les éditions de la Maison des sciences de l'Homme

Édition imprimée

Date de publication : 5 octobre 2017

Pagination : 13-27

\section{Référence électronique}

Pierre France et Alessio Motta, «En un combat douteux. », Quaderni [En ligne], 94 | Automne 2017, mis en ligne le 05 octobre 2019, consulté le 23 juillet 2021. URL : http://journals.openedition.org/quaderni/ 1103 ; DOI : https://doi.org/10.4000/quaderni.1103 


\section{$D$ ossier}

En un combat douteux. Militantisme en ligne, «complotisme» et disqualification médiatique : le cas de l'association ReOpen911

\section{Pierre}

France

Doctorant en Science Politique ATER Sciences Po, Aix-en-Provence Université Paris I Panthéon Sorbonne CESSP-CRPS

\section{Alessio Motta}

\author{
Doctorant en Science Politique \\ ATER Université Paris I \\ Panthéon Sorbonne \\ CESSP-CRPS
}

Bien que le « complotisme » soit devenu un marronnier journalistique et suscite ces dernières années de multiples travaux scientifiques, rares sont les approches proprement sociologiques du sujet. Présenté comme un vague "phénomène de société », traité surtout par des « théoriciens médiatiques », le complotisme semble offrir peu de prise à un travail de terrain. L'objectif de cet article est de combler ce vide en traitant de l'ordinaire du " complotisme » avec les outils ordinaires des sciences sociales. Les pages qui suivent s'appuient sur une recherche commencée en 2014 sur l'association ReOpen911 qui se présente comme un mouvement visant à «informer les citoyens sur les attentats du 11septembre 2001, cuvrer pour l'établissement d'un débat public, et militer pour l'ouverture d'une enquête approfondie sur cette tragédie ${ }^{l} »$.

L'enjeu est à la fois de proposer de premiers éléments pour un travail sur les profils sociaux des " complotistes », et non plus seulement sur leur profil psychologique ou leurs pathologies ${ }^{2}$, mais aussi d'offrir une vue processuelle de l'évolution de l'association et des carrières de ses membres. Plutôt que de nous focaliser sur les thèses complotistes elles-mêmes, nous aborderons la création et l'attribution du stigmate " complotiste », qui s'inscrit dans des interactions. Nous présenterons ces dynamiques en trois temps. Tout d'abord, nous évoquerons la création de l'association ReOpen911 en 2006, son ancrage à gauche et ses liens avec les outils de communication en ligne qui se développent alors. Puis nous aborderons les clivages internes et le travail de distinction opéré par certains de ses membres face aux tentatives d'appropriation du mouvement par des organisations d'extrême droite, à partir de la 
fin des années 2000. Enfin, nous verrons le lien que l'association entretien avec la démarche de critique des médias et l'effet structurant de leurs interactions avec les journalistes sur la création d'un stigmate du complotisme.

Une telle approche pose plusieurs défis. D'abord, il n'est pas question d'exposer ici le contenu des différentes versions du 11 septembre qui circulent, ce même si les théories défendues par tels ou tels membres de ReOpen911 sont en lien avec leur position - au centre ou en marge - dans l'association, avec différents bords politiques et plus globalement avec leur position et origines sociales. Ensuite, l'approche constructiviste et interactionniste pour laquelle nous avons opté apparaît d'autant plus délicate à manier qu'elle est aujourd'hui mobilisée implicitement ou explicitement par certains truthers (militant mettant en cause les versions couramment admises des attentats du 11 septembre) $)^{3}$. L'idée et le discours selon lesquels le complotisme est un stigmate utilisé stratégiquement pour délégitimer ${ }^{4}$ ont été intégrés par nos enquêtés ${ }^{5}$, et renvoyé à leurs adversaires. Le risque est grand d'apparaître comme ceux qui les aident, volontairement ou non, à construire un discours de re-légitimation, et d'apparaitre trop « compréhensifs ${ }^{6} »$. À l'inverse, un autre risque est de se contenter de répéter les discours critiques à l'égard de l'association. La posture adoptée ici s'appuie sur une tradition de recherche, visant à aborder les objets les plus illégitimes ou dont les chercheurs se sentent moralement ou politiquement éloignés, sans avoir recours à des grilles de lectures exceptionnalistes qui sortent du domaine des sciences sociales, et sans s'aventurer dans une lecture pathologisante, misérabiliste ou bien fonctionnaliste ${ }^{7}$. Ce que cet article entend développer a bien plus à voir avec l'ordinaire de la sociologie interactionniste et de celle du militantisme et permettra de reconstituer l'espace de contestation autour du sujet du 11 septembre $^{8}$ (qui dépasse la seule association $\mathrm{ReO}-$ pen911) et le tissu d'interactions dans lesquelles l'association et ses membres sont pris.

Nous nous appuyons en premier lieu sur une observation d'événements (deux assemblées générales, une conférence) et sur des entretiens réalisés avec 15 membres, anciens membres et sympathisants du mouvement ${ }^{9}$. Si certains auteurs évoquent un " phénomène complotiste » qui serait le « reflet d'une époque ${ }^{10} »$, d'autant plus inquiétant qu'il paraît impossible à incarner, ReOpen911 présente la particularité d'être une organisation clairement identifiable. L'organisation laisse de nombreuses traces sur Internet, nous laissant la possibilité d'étudier le contenu de son site $^{11}$, son forum en ligne, sa page Facebook, et son activisme sur d'autres sites. Plusieurs de ses membres interviennent par exemple sur le fil - encore actif - consacré au 11 septembre du forum très fréquenté de Hardware.fr. Le forum de ReOpen911 s'est avéré un outil précieux pour observer des interactions directes entre membres à différents moments de l'existence de l'association, ou les présentations qu'ils font d'eux-mêmes. Il a été pour nous ce qu'il est aussi au fond pour bien des membres de l'association (mais dans leur cas pour y trouver des informations sur le 11 septembre) : un centre d'archives.

Les références au forum en entretien s'accompagnaient souvent d'une mise en garde : ce n'est « pas le site » et il a « failli être fermé à plusieurs reprises ». Son contenu, même largement modéré, 
laisse en effet entrevoir l'envers d'un site et d'une ligne mise en avant par l'association. On y trouve les signes des dissensions au sein de l'association, les différences de vues entre les sympathisants et le noyau dur des membres. C'est aussi là que sont avancées le plus de théories " différentes » ou « marginales » et que l'on déborde potentiellement le cadre des «points clés » sur le 11 septembre ${ }^{12}$ et la neutralité que l'association affiche sur son site.

Nous avons aussi procédé à une recherche de données sur les pages Internet liées à ReOpen911, qu'il s'agisse des pages citées sur le site de l'association ou qui renvoient vers ce dernier de manière positive ou critique. Car l'association entretient des rapports conflictuels avec les médias en général, mais aussi avec différents acteurs et organisations en particulier. On relève notamment des adversaires pour le monopole du discours contestataire sur le 11 septembre (comme Égalité et Réconciliation ${ }^{13}$ ), mais aussi des adversaires « anti-complotistes », comme Conspiracy Watch $^{14}$ : c'est en ce sens que nous avons recours à la notion d' « espace » de contestation. Nous avons exploré l'écosystème de sites, blogs (sur le 11 septembre ou d'autres sujets) et pages personnelles (sur des sites comme LinkedIn ou Facebook) de certains membres. Toute cette matière n'implique pas pour autant un accès total à la vie de ReOpen911. Non seulement le forum n'a pas été entièrement lu dans sa version accessible à tous les inscrits en ligne, mais il comporte des parties cachées réservées aux membres de l'association. Celles-ci sont restées hors de notre portée, de même que les « messages privés » et échanges réalisés via d'autres moyens de communication ${ }^{15}$.

\section{Une association « citoyenne » d'information sur le 11 septembre 2001}

ReOpen911 ne se développe pas en 2001 à la suite d'un « choc moral » ou d'une « sidération » post-attentat ${ }^{16}$, ni même suite à la publication en 2002 de l'ouvrage de Thierry Meyssan sur le 11 septembre ${ }^{17}$. Le site est d'abord le résultat d'un buzz provoqué par la traduction de la vidéo Loose Change en français, début 2006. Premier « blockbuster Youtube $»^{18}$, Loose Change, autoproduit par un étudiant américain, popularise la thèse de la « démolition contrôlée », selon laquelle les tours du World Trade Center auraient été chargées d'explosifs avant les crashs. C'est largement autour de cette thèse que se lance le Truth Movement américain, mouvement de contestation autour du 11 septembre. Bien plus que le livre de Thierry Meyssan, ce film est cité par plusieurs anciens membres de ReOpen911 comme une source décisive de leur engagement. L'artisan de cette traduction, Atmoh, crée ReOpen911.info sous forme de blog dans la foulée, avec pour vocation de continuer ce «travail citoyen de traduction et de mise à disposition d'informations », centré sur les vidéos (en mars 2006, 11 vidéos sont déjà partagées sur le site). Si ReOpen911 emprunte son nom à un site américain, créé dans le même contexte (reopen911.org), il n'en est pas une antenne, pas plus que ses membres n'entretiennent en réalité de liens directs avec les truthers américains. À une exception près, aucun de nos enquêtés n'a été aux États-Unis, et les contacts sont peu nombreux entre ces deux mouvements qui se construisent largement en autonomie, d'autant plus que parmi les membres de ReOpen911 seul un petit noyau dur maîtrise l'anglais. En revanche, le mouvement étudié a une échelle 
francophone (le site compte des membres suisses, belges ou québecois) et constitue l'unique organisation de cette taille en Europe.

Au plus fort de son audience, en 2006-2007, le site connaît un succès notable avec environ 4000 à 5000 visites par jour, et ReOpen911 devient rapidement plus qu'un blog. À la fois site, forum, association, organisateur de conférences et de projections, ReOpen911 est un ensemble à géométrie variable, entre Internet et militantisme traditionnel, entre Paris et petits groupes en province, entre travail de réflexion et création de théories et travail de diffusion.

L'activité de l'association se situe à la croisée du journalisme citoyen, de l'altermondialisme, mais aussi des communautés d'amateurs sur Internet, beaucoup moins politiques. ReOpen911 se rapproche d'un mouvement de $«$ fansubs $»^{19}$ par sa dimension d' « équipe de traduction », tout autant qu'il fait activement référence à l'utilisation d'un peer to peer alors dans ses heures de gloire, et exalte le « pouvoir d'Internet»: "Loose Change c'est arrivé avec le haut-débit, la première fois qu'on pouvait regarder un film d'1h30 $30^{20}$. La figure de l'amateur connecté ${ }^{1}$ propre à ce réseau semble du reste largement correspondre à l'auto-présentation de plusieurs membres qui, parfois par leur emploi, souvent par leurs loisirs et disponibilité biographique, passent des heures quotidiennes en ligne. Dans ce groupe largement masculin ${ }^{22}$, plusieurs sont « ingé-son », graphistes, traducteurs, vidéastes, informaticiens de fait, sans avoir forcément les diplômes correspondants ni travailler spécifiquement dans le domaine qu'ils annoncent. Plusieurs n'ont pas trente ans en 2006-2008, ne sont pas stables professionnellement ou n'ont pas tout à fait terminé leurs études. Le mouvement compte certes des diplômés et des professionnels de secteurs dont il est question dans les débats techniques sur le 11 septembre (parmi nos interlocuteurs, on relève par exemple deux ingénieurs et un physicien) et communique souvent sur la présence d'architectes et de scientifiques dans ses rangs ${ }^{23}$, mais il est surtout le fait de ces $a m a-$ teurs, pour lesquels ReOpen911 est un espace d'apprentissage, et de « travail » professionnel et politique. L'engagement dans ce mouvement marque pour certains le début d'une carrière (de traducteur ou de vidéaste dans plusieurs cas), voire une occasion de changer de voie (l'un des enquêtés cite ainsi son passage par ReOpen911 sur sa page LinkedIn comme une expérience professionnelle). Par ailleurs, pour tous ou presque, c'est l'occasion d'un premier engagement, impliquant apprentissage du militantisme et acquisition de compétences politiques, pour "s'intéresser au monde moderne » au-delà du 11 septembre.

Si cet engagement est aujourd'hui vécu comme difficile et coûteux, être sympathisant ou membre actif de ReOpen911 paraissait initialement plus anodin et semblait s'inscrire dans la continuité d'autres mobilisations à gauche. L'illégitimité de cette association dans l'espace militant n'est pas immédiate : aucun des premiers membres ne voit là un engagement radical ou extrémiste. ReOpen911 appartient au départ aux queues de comète de l'altermondialisme, auquel elle emprunte un usage important d'Internet ${ }^{24}$. Elle se présente aussi sous l'apparence de bien d'autres mouvements « monothématiques » (Sortir du nucléaire, Jeudi noir, Génération précaires, etc. ${ }^{25}$ de l'époque. Les parcours des membres de 
ReOpen911 rencontrés confirment cet ancrage : ils sont sympathisants $\mathrm{du}$ " non » au référendum de 2005 sur le traité constitutionnel européen, citent ATTAC, le NPA, des associations ou des syndicats. ReOpen911 cherche d'abord explicitement à se rapprocher de phares de la gauche comme interlocuteurs et emprunte au militantisme le plus classique ses répertoires d'actions. À rebours de la théorie d'un complotisme qui se déploierait avant tout sur internet, pendant les premières années, l'activité de ReOpen911 repose au moins autant sur le militantisme « traditionnel » que sur l'activité en ligne. Les militants diffusent des tracts dans les lieux publics, à la Fête de l'Humanité, devant une projection d'un film de Naomi Klein, prennent contact avec des élus, et surtout organisent des projections de films. Ils se joignent aussi à des événements liés à la guerre en Irak, en plaçant ce qu'ils espèrent être le futur scandale du 11 septembre dans la continuité de ceux des « armes de destruction massive » ou des tortures à Guantanamo. Ce militantisme est alors peu hiérarchisé et contrôlé, de sorte que l'activité de ReOpen911 est très largement décentralisée, et apparaît comme une marque que chacun s'approprie ${ }^{26}$. Il est aussi le fait d'une toute petite minorité très active et pour laquelle l'expérience a souvent été intense ${ }^{27}$. Les 10000 inscrits sur le forum et les 34000 « likes » jusqu'à aujourd'hui sur Facebook masquent bien entendu une nette différence entre le noyau dur militant et les simples passants sur le net. Pour preuve, l'association, qui regroupe une grande part de ces militants les plus actifs, n'a compté au maximum que 160 membres. Se détache nettement une " élite » réduite de membres organisateurs des événements, militants sur le terrain, traducteurs, et contributeurs aux discussions de fond sur les théories, dans laquelle on retrouve ceux qui se rapprochent le plus des profils socialement dominants (ingénieur ou avocat, par exemple). Si le forum et le site forment un espace de stockage des informations et traductions et un « espace de travail », ils sont aussi un espace de distinction où les plus valorisés sont ceux qui finissent par avoir leur propre théorie ou leur spécialité d'analyse reconnue.

\section{Expropriée à gauche et appropriée par l'extrême droite, une association sur le fil}

Le passé de gauche de ReOpen911 est toutefois assez peu visible et n'est jamais mis en avant par l'organisation, largement mise en cause depuis pour des liens avec l'extrême droite. L'association affiche une neutralité sur les causes de l'effondrement des tours, sans accusation ciblée en se contentant de demander " l'ouverture d'une nouvelle enquête ». Elle affiche enfin une neutralité politique, "poser des questions sans pour autant apporter des réponses, c'est la ligne qu'on a tenue à $\mathrm{ReOpen}^{28}$ » est une idée qui revient souvent en entretien, et que le site met activement en scène. Difficile à première vue de situer l'organisation et les membres, pour beaucoup attachés à l'étiquette de « citoyen absolument ordinaire [ne militant] pour aucun parti, aucun mouvement politique et aucune idéologie ».

Pourtant cette neutralité est moins un véritable choix politique (ou un faux-semblant qui cacherait un ancrage plus clair) qu'un moyen de neutraliser les divisions dans un mouvement jeune, à l'organisation fragile, et aux orientations politiques rapidement divergentes. Elle est le reflet d'une situation où le mouvement est à la fois exproprié 
sur sa gauche - notamment sous le coup d'une accusation d'antisémitisme - et confronté à des tentatives d'entrisme ou de dépassement par la droite, qui sont aussi le reflet de nouveaux arrivants au-delà du noyau fondateur.

Dès 2006, les membres sont invités dans les règles d'utilisation «à relativiser les théories extravagantes, ne pas parler d'extra-terrestres ou de tout hors-sujet susceptible de décrédibiliser votre argumentaire ». Mais c'est l'accusation d'antisémitisme qui va très vite être la plus lourde à gérer pour le mouvement, et amener de nombreuses organisations de gauche à rompre tout lien avec lui et avoir des conséquences sur la vie des membres («Plus moyen d'en parler, on en perd ses amis ou ses proches. Assimilé à des extrémistes ou à un antisémite »). Conspiracy Watch relève à partir de 2007 certains passages sur le forum, ou souligne des liens ou des propos antisémites ${ }^{29}$. Une accusation vécue comme « injuste » par les principaux membres de l'époque, qui répondent surtout en accusant en retour leurs adversaires d'être « néo-conservateurs ». La gestion de ce stigmate va à partir de ce moment-là rythmer de manière importante la vie de l'organisation, qui tente de se prémunir d'autres accusations en procédant à la mise en place d'une modération plus lourde sur le forum, ou l'ouverture d'une partie cachée où sont renvoyées les discussions sur le sujet. Graduellement, certaines figures comme celle de Thierry Meyssan sont aussi délaissées sur le site, les publications de son Réseau Voltaire cessent ainsi d'être relayées à partir de 2009.

Quelques années après sa création, ReOpen911 se trouve divisée entre plusieurs lignes politiques, et confrontée à la concurrence ou au soutien d'autres organisations qui cherchent à s'emparer du sujet du 11 septembre. Fraichement accueillie à gauche à la suite des accusations évoquées plus haut, l'association est par contre bienvenue dans d'autres mouvements comme Égalité et Réconciliation. À partir de 2011, le site d'Alain Soral évoque de plus en plus fréquemment le sujet du 11 septembre, avec une stratégie explicite de récupération de possibles adhérents ${ }^{30}$. Pour les 10 ans de l'événement, $E \& R$ publie ainsi un livre, organise une conférence sur le sujet, et se fend d'articles élogieux sur ReOpen911, tout en ayant à disposition sur le forum un certain nombre de relais. En réaction, ReOpen911 invite certains membres entretenant des liens avec $E \& R$ à s'éloigner de l'association. Une potentielle demande à adresser au site d'Alain Soral pour retirer le site de ses liens est longuement étudiée par des responsables de l'association, mais finalement elle n'est jamais envoyée ${ }^{31}$.

Le tiraillement autour de ces questions se joue indirectement dans le contenu des théories sur le 11 septembre discutées en ligne. L'entrisme d'extrême droite s'accompagne de la promotion de théories alternatives... aux théories alternatives les plus courantes chez ReOpen911. L'association voit se retourner contre elle les critiques qu'elle a adressée aux journalistes : elle est accusée de ne pas respecter son discours de neutralité et d'ouverture, notamment par des contributeurs du forum qui dénoncent la mise au ban de certaines propositions et l'emprise de «VO spécial truthers » (comprendre versions officielles de ceux qui critiquent la « version officielle » des événements du 11 septembre $^{32}$ ). Plusieurs gros contributeurs exerçant cette dénonciation se 
font les promoteurs d'une théorie ouvertement antisémite et/ou d'une autre, celle du « no plane » (selon laquelle aucun avion n'aurait frappé le World Trade Center), conçue comme un nouveau paradigme rendant caduc tout le travail cumulatif sur les autres théories précédentes ${ }^{33}$. Enfin, plus globalement ils en appellent régulièrement à étudier d'autres événements pour dépasser le sujet du 11 septembre et reprendre prise avec l'actualité : révolutions arabes, situation en Ukraine, attentats en France... Une démarche de rebond sur l'actualité qui, aux yeux des principaux responsables de ReOpen911, correspond trop au « complotisme », autre étiquette dont il devient d'année en année plus important de se distancier. L'association préfère revendiquer un recentrage sur le 11 septembre.

Mais l'antisémitisme à ReOpen911 ne saurait se résumer (comme il nous l'a été rapporté en entretien) à un problème d'entrisme émanant d'autres organisations, pas plus qu'il n'est une labellisation « injuste » par des acteurs extérieurs utilisant des débordements isolés pour décrédibiliser le mouvement dans son ensemble. Il est plutôt le reflet d'une organisation peu centralisée, « mouvement citoyen » qui cherche avant tout dans ses premières années à recruter un maximum de soutiens, et s'avère surtout investi par des personnes novices en politique, pour lesquelles le clivage gauche/droite ou la gravité de l'accusation d'antisémitisme n'ont au départ rien d'évident. Beaucoup manifestent une " incompréhension » et une " surprise » à l'époque vis-à-vis des conséquences que leur engagement a pu avoir et des accusations dont ils ont fait l'objet. En ce sens, l'étude du complotisme a autant à voir avec les études sur l'ex- trémisme que celles sur la compétence politique et les primo-militants.

En tout état de cause, les clivages et le caractère d' « auberge espagnole » de ReOpen911, mêlés aux prises de positions de ses principaux responsables, débouchent sur une situation paradoxale. Alors qu'à partir de 2013, les articles émanant d' $E \& R$ finissent par devenir hostiles et voient dans ReOpen911 un « idiot utile », une « opposition contrôlée » qui pose certaines questions tout en en refusant d'autres, l'association est au même moment encore mise en cause à gauche. Jugée proche de l'extrême droite dans les marronniers médiatiques sur le « complotisme » et le « confusionnisme », elle devient la cible de mouvements antifascistes. Deux enquêtés racontent que, dans leurs régions respectives, ces derniers ont dénoncé, perturbé et fait annuler des événements de ReOpen911, tandis que d'autres ont vu leur identité dévoilée par un article sur un site de la mouvance.

\section{« Journalistes contre citoyens »}

D'association dont les membres étudient et publient sur le sujet du 11 septembre, ReOpen911 est aujourd'hui surtout devenu un mouvement qui passe l'essentiel de son temps à réfuter et gérer les étiquettes d'antisémite et - de plus en plus de « complotiste » qu'on lui accole. Plus que des discussions sur le 11 septembre, nos entretiens sont l'occasion de longs développements sur le pouvoir de la propagande et de médias auxquels on attribue la responsabilité de ce stigmate. Tandis qu'un membre évoque son indignation face à un documentaire anti-complotiste de 2004 - présenté comme le déclic de son intérêt pour le 
11 septembre - d'autres parlent de leur colère à la suite d'un reportage sur le mouvement en avril 2008, jugé injuste au point que plusieurs se sont lancés dans la réalisation d'un contre-documentaire. Aujourd'hui, le site chronique ses « droits de réponse » envoyés régulièrement à tous les journaux qui évoquent l'association.

D'emblée pensé comme un « site d'information » en 2006 avec une «partie news », ReOpen911 se veut un outil de « journalisme citoyen », proche d'Agoravox (alors l'un des principaux sites de journalisme participatif, sur lequel le mouvement et plusieurs membres publient), et en lien avec un écosystème de blogs (à commencer par celui du fondateur Atmoh). C'est aussi un agrégateur de contenus, où sont repris divers articles de journaux.

Fondamentalement, l'organisation s'est donc construite depuis sa création comme et contre les médias, dans un dialogue constant avec eux. Mais initialement, elle ne cherche pas à se substituer à ces derniers, dont elle espère un « déclic » qui permettra l'éclatement d'un scandale « 11 septembre ». Le mouvement sollicite même en permanence divers médias (en témoignent les longues listes de mails de journalistes qui s'échangent au début sur le forum). De grands espoirs sont placés dans un journalisme de gauche, auprès de Daniel Mermet, l'association Acrimed $^{34}$, ou Serge Halimi ${ }^{35}$. Espoirs rapidement déçus : fin 2006, Le Monde diplomatique publie un article du journaliste Alexander Cockburn reprochant à des pans de la gauche américaine son aveuglement à penser les événements du 11 septembre avec les yeux des droites populiste et libertarienne. Comme le souligne l'un des pre- miers membres de ReOpen911, « [À l'époque,] on est à mille lieux de penser que ça va être attaqué. On est naïfs, on pensait ouvrir un débat à gauche, avec des alliés politiques objectifs. En 2007, il y a cet article dans Le Monde diplo, c'est le pire de [son] histoire [...] On est très vite taxés d'antisémitisme. $[\ldots]^{36}$ ».

Jusque-là, les membres de l'association voyaient dans les réticences des journalistes une dimension réversible, masquant un phénomène souterrain dont ils relevaient méthodiquement les « brèches » médiatiques : un enquêté évoque ainsi « tous ceux qui ont acheté le dvd à l'époque, la liste est étonnante! ! ${ }^{37} »$, un autre relate une rencontre où le journaliste «fuit prudemment parce qu'on était en public » (sous-entendant qu'il n'en pense pas moins), certains rappellent des parutions et contacts médiatiques soulignant l'intérêt de leur travail (contacts avec Denis Robert, Karl Zéro, ou bien article favorable dans l'édition norvégienne du Monde diplomatique).

Potentiels « alliés objectifs » au début, les journalistes sont peu à peu considérés comme un problème à part entière et un des facteurs de l'échec des truthers. De fait, le « cœur de métier» de l'association change peu à peu. En 2016, le débat est moins centré sur les attentats du 11 septembre que sur le traitement médiatique dont les événements, ReOpen911 et le « complotisme » ont fait l'objet. On y trouve ainsi les traces des multiples interactions que les membres de ReOpen911 entretiennent avec les médias et, partant, d'un mécanisme de construction et d'entretien d'un stigmate « complotiste ».

De façon générale, le traitement médiatique dont 
ReOpen911 fait l'objet, loin de reposer seulement sur les thèses promues par ses membres, résulte des rapports quotidiens progressivement entretenus par les membres avec les journalistes. Une partie des membres les plus actifs sur la partie « news » du site revendiquent leur égalité avec ces derniers, et leur capacité à écrire eux aussi des articles ou à réaliser des documentaires. D'autres font des newsletters destinées à leurs familles ou $\mathrm{amis}^{38}$. Les interactions s'en trouvent souvent retournées : le mouvement oppose aux journalistes leurs propres règles (charte de Munich, conditions d'utilisation des commentaires sur les sites) et leurs propres pratiques, à l'image de cet enquêté étonné de s'être vu refuser l'enregistrement d'un entretien par un journaliste : «je passe pour parano parce que j'enregistre la discussion ! ${ }^{39} »$. En 2009, les journalistes de « complément d'enquête » sont filmés par ReOpen911 en train d'interviewer quatre membres, et ensuite mis en cause par ce biais pour leur montage partial. Plus encore, les membres de ReOpen911 sollicitent régulièrement les journalistes pour les interviewer à leur tour, ou bien réagir à ce qu'ils ont pu écrire.

En entretien, plusieurs membres décrivent les réactions de journalistes qui «perdent les pédales pour s'enfermer dans une attitude ordurière de rejet », ne répondent pas ou bien coupent court aux échanges. Sans avoir besoin de mettre en cause le contenu des théories avancées par ReOpen911 dans ces interactions ${ }^{40}$, il est vraisemblable que la manière de faire de ces militants heurte l'ordinaire professionnel de journalistes plus habitués à solliciter leurs sources qu'à l'inverse ; formés à le faire dans un temps limité et non, comme certains membres de ReOpen911, « en passant des jours à tout lire sur internet $»$. À travers des mailing et avalanches de commentaires dans le moindre article sur le 11 septembre $^{41}$; la présence systématique dans des réunions publiques; ou bien les tentatives de discussions à la volée dans divers contextes, l'association est très présente jusqu'à être accusée de harcèlement ${ }^{42}$ et susciter des réactions vives de la part de journalistes : " l'année dernière on avait fait une manif devant les médias pour l'anniv du 11/9, on était devant le Nouvel Obs, Joffrin descend et fume une clope. [...] Il y a eu un échange [...] ça a tellement énervé Joffrin que le lendemain il a publié quelque chose 'les complotistes ennemis de la démocratie ${ }^{43} »$.

La situation n'a en soi rien d'inédit pour peu que l'on ait en tête la « crise du journalisme » dans les années 2000, où le rapport avec le lecteur est justement en question ${ }^{44}$. Ce refus par des journalistes enquêteurs de devenir les enquêtés des autres se retrouve dans bien d'autres situations ${ }^{45}$, tout autant que la difficulté, au-delà du champ journalistique seul, à accepter pour des professionnels de donner une place à des profanes ${ }^{46}$. Elle a cependant dans notre cas un caractère itératif : en cherchant à se débarrasser des accusations dont ils font l'objet, les membres de ReOpen911 alimentent continuellement un mécanisme qui les rend illégitimes. Il est aussi probable, même si une enquête en ce sens resterait à faire, qu'il n'est pas non plus possible du point de vue des journalistes d'appréhender le complotisme comme un sujet ordinaire ou vierge, en évitant toute démarche de distinction et de critique ${ }^{47}$.

L'incapacité de ReOpen911 à mobiliser les médias dans le sens attendu est vraisemblablement pour beaucoup dans le relatif abandon qui touche 
l'association sur la dernière période. Alors qu'ils ont baigné, les premières années, dans la prophétie de l'éclatement imminent d'un scandale public sur les « zones d'ombre » du 11 septembre ${ }^{48}-$ dont ils ont cherché les signes annonciateurs en espérant un effet boule de neige ( « [avec le 9/11] démonter un seul point de façon certaine, c'est tout démonter du même coup ») - celle-ci ne s'est pas réalisée ${ }^{49}$. Au moment où nous commençons à étudier l'association, elle apparaît désertée et beaucoup de membres s'en sont éloignés, «En 2006-2011, on espérait... Maintenant je suis plus trop optimiste. [...] J'ai travaillé dessus pendant cinq ans, il faut savoir passer à autre chose ${ }^{50}$ ».

\section{Conclusion : le " complotisme », prophétie autoréalisatrice ? Remarques sur le traitement médiatique d'un problème public}

Cette première restitution d'enquête tend à souligner que le « complotisme » est loin d'être une idéologie unifiée et sans histoire. Il est avant tout un label, une étiquette au sens interactionniste. Non pas parce qu'il résulterait d'une attribution autoritaire par des acteurs critiques du mouvement, mais parce que l'attribution d'un tel label à des individus ou à une organisation doit parfois moins aux thèses que ceux-ci défendent qu'aux diverses interactions dans lesquelles ils s'engagent. La relative inexpérience politique des militants de ReOpen911, le choix d'une organisation « ouverte » dans un espace de contestation où l'on croise rapidement des acteurs d'extrême droite et/ ou antisémites, et enfin et surtout leur stratégie d'échange avec les journalistes ont joué un rôle déterminant dans la construction et l'entretien du stigmate « complotiste». Ce stigmate affecte les membres de ReOpen911, et au-delà sa présence médiatique croissante a contribué depuis le milieu des années 2010, à construire les théories du complot comme un danger nécessitant la mise en œuvre de stratégies de la part des journalistes, et même de politiques publiques ${ }^{51}$.

Pourtant, peu de données attestent solidement du caractère massif du phénomène. La désertion dont souffrent ReOpen911 et son forum sur les dernières années ne plaide pas en ce sens ${ }^{52}$. Il est plus adéquat d'évoquer un flou sur l'audience des thèses complotistes, fortement lié à leur diffusion sur Internet et aux incertitudes techniques relatives à l'audience sur ce média. Un flou lié aussi à l'hétérogénéité que cache le terme valise de « complotiste » : il est difficile de faire la distinction entre ceux qui ont « quelques doutes » et ceux qui croient à des méta-complots, entre ceux qui consomment et ceux qui inventent des théories concernant un événement précis. Tout comme il est difficile de faire la différence entre ceux qui consultent les sites complotistes par sympathie, par curiosité ou par dérision et dont les clics sont indifférenciés. Plus encore, la surévaluation du complotisme résulte aussi d'un intérêt partagé par tous les acteurs de cet espace, qui mobilisent les mêmes outils sondagiers et informations parcellaires sur l'ampleur de la chose : pour 58\% des Français qui croient à la théorie du complot chez ReOpen911, ils sont 45\% sur le site Spicee $^{53}$. Cette surévaluation fournit aux organisations complotistes un moyen de légitimation, et offre parallèlement aux intellectuels et journalistes qui les critiquent un outil de dramatisation ou de plaisanterie.

Tout comme on a pu se demander si la mise en cause des journalistes n'a pas, pour ReOpen911, 
un effet pervers, on est au final fondé à se demander dans quelle mesure la dénonciation médiatique des théories du complot soulève ou règle un problème et dans quelle mesure elle le crée en diffusant ces théories, même avec une intention ouvertement critique. L'un de nos enquêtés qui dit s'intéresser à diverses théories du complot admet d'ailleurs qu'il puise une partie de ses informations et de son inspiration sur le site Conspiracy Watch, sur lequel sont pourtant dénoncées ces théories : «c'est très pratique [...], j'y vais souvent! »
$\mathrm{N} \cdot \mathrm{O} \cdot \mathrm{T} \cdot \mathrm{E} \cdot \mathrm{S}$

1. http://www.reopen911.info/, consulté le 13 février 2017.

2. Cf. par exemple Bronner (G.), La démocratie des crédules, Paris, PUF, 2013 ; ou plus ancien Graumann (C.F). et Moscovici (S.) (dir.), Changing Conceptions of Conspiracy, New York, Springer, 1987.

3. Un truther et sociologue américain a écrit une thèse sur le sujet. Ellefritz (R.), Discourse Among the Truthers and Deniers of 9/11: Movement-Contermovement Dynamics and the Discursive Field of the 9/11 Truth Movement, Thèse de philosophie, Oklahoma State University, 2014.

4. Maler (H.), Champagne (P.), « Usages médiatiques d'une critique "savante" de "la théorie du complot" ", Agone, 2012, pp. 167-178.

5 . On retrouve ici une dynamique classique de « double herméneutique ». Giddens (A.), La constitution de la société : Eléments de la théorie de la structuration, Paris, Presses Universitaires de France - PUF, 2012.

6. Bizeul (D.), « Des loyautés incompatibles. Aspects moraux d'une immersion au Front National », SociologieS [en ligne], mis en ligne le 21 juin 2007.

7. Posture dont les jalons sont posés dans Aldrin (P.), «Penser la rumeur. Une question discutée des sciences sociales », Genèses, 2003, 50 (1), pp. 126-141 ; Pour de premières tentatives d'application à la question du complotisme cf. France (P.), Pour une sociologie politique du complot(isme), Working Paper CESSP, 2015 ; et Motta (A.), « Mépris et répression de la prise de parole en public. Construction d'une domination symbolique profane dans une copropriété et dénonciation publique », Participations, 2014, 2 (9), pp. 71-95.

8. Mathieu (L.), L'espace des mouvements sociaux, Bellecombe-en-Bauges, Le croquant, 2012. 
9. Auxquels nous posions des questions entre autres sur leur arrivée à ReOpen911, leurs activités au sein du mouvement, leurs professions, diplômes et origines sociales, et leur rapport au politique.

10. Taguieff (P.-A.), L'imaginaire du complot mondial : Aspects d'un mythe moderne, Paris, Mille et une nuits, 2006.

11. Y compris avec une profondeur temporelle par le recours à the internet archive où le site de ReOpen911 a été capturé 380 fois depuis 2006.

\section{2. http://www.reopen911.info/points-11-septembre/1.} html, consulté le 13 février 2017.

13. Mouvement fondé en 2007 par Alain Soral et des anciens militants du mouvement étudiant Groupe Union Défense (GUD), et qui cherche explicitement à se positionner entre extrême droite et extrême gauche. 14. «Observatoire du conspirationnisme et des théories du complot », blog dans la lignée d'autres sites comme Hoaxbuster, fondé en 2007, qui recense et dénonce les théories du complot. Il est devenu la principale référence « anti-complotiste » depuis quelques années. 15. Nous ferons des rappels aux entretiens tout au long de l'article en note de bas de page. Par souci de place, le reste des références entre guillemets, provenant $d u$ site ou du forum, ne fera pas l'objet d'un référencement aussi systématique.

16. De manière notable, personne au sein de ReOpen911 ne se rappelle avoir eu un choc le jour même du 11 septembre, à l'image de cet enquêté : « Le jour du 11 septembre j'ai tout gobé force 12 ! Si j'étais pas tombé sur ce tract, qui sait, je serais encore néophyte ! » (entretien, 7/06/2016). Sur les réactions post-attentat cf. Truc (G.), Sidérations. Une sociologie des attentats, Paris, PUF, 2016.

17. L'ouvrage de Thierry Meyssan, L'Effroyable imposture, qui le premier avance une thèse « complotiste » sur le 11 septembre, lorsqu'il est publié début 2002, déclenche un scandale médiatique doublé d'un phénomène d'édition qui aura un aspect très structurant sur l'appréhension du problème du complotisme en France.

18. « Click Here for Conspiracy », Vanity Fair, août 2006. http://www.vanityfair.com/news/2006/08/ loosechange200608, consulté le 10 mai 2017

19. Dagiral (E.), Tessier (L.), « 24 heures ! Le soustitrage des nouvelles séries télévisées », in Gaudez (F.), dir., Les arts moyens aujourd'hui, Paris, l'Harmattan, 2008, p. 107-123.

20. Entretien, 14/06/2016.

21. Flichy (P.), Le Sacre de l'amateur: Sociologie des passions ordinaires à l'ère numérique, Seuil, 2014.

22. Sans présager d'identités laissées volontairement floues par l'usage de pseudonymes sur internet, nous n'avons pu identifier que quatre femmes qui apparaissent importantes dans la vie de l'association entre 2005 et aujourd'hui.

23. Voir par exemple l'interview dans Cnews. http:// www.cnewsmatin.fr/monde/2013-05-29/arnaudberard-ae911-compte-des-dizaines-dingenieursstructure-474572, consulté le 13 février 2017

24. Sommier (I.), Fillieule (O.), et Agrikoliansky (E.) (dir), Généalogie des mouvements altermondialistes en Europe: Une perspective comparée, Paris : Aixen-Provence, Karthala, 2008 ; Granjon (F.), Cardon (D.), « Les mobilisations informationnelles dans le mouvement altermondialiste ", Colloque "Les mobilisations altermondialistes », 2003.

25. La comparaison se justifie d'autant plus que l'un des fondateurs de ReOpen911 a participé à la création de Jeudi Noir.

26. Les groupes locaux ont leurs blogs, leurs propres tracts, etc.

27. Au point que les références au coût d'un tel engagement sur la vie de couple ou les relations amicales font jusqu'à l'objet de blagues, à l'image de l'introduction d'un conférencier américain dans un 
événement organisé par l'association : «Vous auriez dû amener vos amis. Oh bien sûr, vous n'en avez pas [rires] [...] Je vais vous donner une raison de les ramener. Ou d'en perdre encore plus ».

28. Entretien, 01/06/2016.

29. Cf. notamment http://www.conspiracywatch.info/ Le-Ministere-de-la-Crise-du-logement-infiltre-par-lesconspirationnistes-de-reopen911_a144.html, consulté le 13 février 2017.

30. Voir Paichard Léo, La diffusion d'une idéologie radicale par un mouvement d'extrême droite. Étude de l'association "Égalité et Réconciliation 》, Mémoire de Master 2, Université Paris 1, 2015. Voir aussi, plus polémique, mais instructif sur la volonté «d'entrisme » de l'association, D'Angelo (R.), Molard (M.), Le Système Soral, Paris, Calmann-Lévy, 2015.

31. Entretien, 21/04/2016.

32. «VO » étant le terme utilisé dans l'association pour désigner péjorativement cette « Version officielle». Parmi les théories alors dominantes, on relève en particulier celle de la « démolition contrôlée ».

33. Au-delà même du contenu de la théorie, cette attitude heurte les règles habituelles chez ReOpen911 qui consistent à s'appuyer sur les travaux précédents : les nouveaux arrivants sont fréquemment invités à utiliser la fonction recherche du forum avant de poster quoi que ce soit. À ce titre, la théorie du «no-plane» bouleverse les hiérarchies du mouvement, où certains sont reconnus comme étant spécialistes de telle ou telle question.

34. Très critique d'un reportage anti-complotiste en 2004. http://www.acrimed.org/Arte-et-la-theorie-ducomplot-une-emission-de-propagande-de-DanielLeconte, consulté le 10 mai 2017

35. Celui-ci, en 2002, renvoyait par exemple dos-à-dos Thierry Meyssan et ses critiques «l'auteur est fondé à ricaner quand, en France [...] des quotidiens qui ont tour à tour gobé les charniers de Timisoara, les bébés de Koweït-Ville jetés hors des couveuses et le plan «fer à cheval » au Kosovo prétendent lui infliger une leçon de journalisme ». Serge Halimi, « Complotite », mai 2002. https://www.monde-diplomatique.fr/2002/05/ HALIMI/8851, consulté le 10 mai 2017.

36. Entretien, 01/06/2016.

37. Entretien, 13/04/2016.

38. «Pour moi, le mieux est l'envoi de courriers, j'ai une centaine de contacts : assos, particuliers [etc.], donc tous les 15 jours, j'envoie un nouveau dossier. » 39. Entretien, 21/04/2016.

40. Dont l'illégitimité tient peut-être partiellement à la disproportion entre la taille des dénonciateurs et celle des forces qu'ils mettent en cause, cf. Boltanski (L.), Darré (Y.), Schiltz (M.-A.), « La dénonciation », Actes de la recherche en sciences sociales, 1984, 51 (1), pp. 3-40.

41. Un article de ReOpen911 évoque « 600 commentaires » sur le site de Marianne. En 2011, les commentaires sont fermés dans un article du Monde sur le sujet.

42. Accusation qui n'est pas limitée à ReOpen911. Voir par exemple, sur l'UPR, http://www.20minutes. fr/elections/presidentielle/2035711-20170325-tentecomprendre-activisme-cybermilitants-upr-francoisasselineau, consulté le 10 mai 2017

43. Entretien, 14/06/2016.

44. Aubert (A.), « Le paradoxe du journalisme participatif », Terrains \& travaux, 2009, pp. 171-190. 45. Bastin (G.), « Le “cas Mathieu” ou l'entretien renversé », Sur le Journalisme / On Journalism / Sobre Jornalismo, 2012, 1 (1), pp. 40-51.

46. Méadel (C.), « Le spectre “psy” réordonné par des parents d'enfant autiste », Politix, 2006, pp. 57-82.

47. Une démarche qui tend d'ailleurs à une montée en généralité vers des enjeux comme la « crise de la démocratie », plutôt que d'être présentée comme ce résultat d'interactions plus banales. Celle-ci illustre 
bien la capacité inégalement répartie à pouvoir monter en généralité entre journalistes et complotistes que nous évoquions plus haut.

48. Non sans rappeler d'autres cas plus anciens. Cf. par exemple Blic (D. de), " "Le scandale financier du siècle, ça ne vous intéresse pas ?’. Difficiles mobilisations autour du Crédit lyonnais », Politix, 2000, 13 (52), pp. 157-181.

49. L'abandon qui en résulte s'oppose ainsi aux conclusions que Leon Festinger tire parfois de ses observations sur le groupe messianique étudié dans Festinger (L.), Riecken (H.), Schachter (S.), L'échec d'une prophétie, Presses Universitaires de France., Paris, 1993.

50. Entretien, 7/06/2016.

51. Cf. notamment la campagne « On te manipule » lancée par le gouvernement en février 2016.

52. Les rencontres physiques sont de moins en moins fréquentées, les débats en ligne s'épuisent aussi. Ce dont les membres et l'association s'inquiètent souvent, à l'image de ce sujet créé sur le forum quelques jours après le 11 septembre 2015, «Que se passe-t-il sur ReOpen ? », qui part du constat de l'absence de la moindre publication pour l'anniversaire de l'événement.: « Le 11 septembre, raison d'exister de l'assos et de notre forum [...], pour la première fois de son existence [...], AUCUN message, AUCUN post ne fut envoyé. [...] Je ne pense pas que nous puissions faire l'économie d'une réflexion sur un fait aussi grave, pourquoi il s'est produit? qu'est ce qu'il sous-tend? [...] il me semble que ça vaut la peine de trouver sinon des solutions immédiates, au moins des raisons objectives de cette absence ».

53. Comme les nombres de visites sur tel ou tel site. Voir aussi http://www.reopen911.info/11-septembre/ sondage-h-e-c-pour-reopen 911-les-francais-et-le11-9-11-questions-sur-le-11-septembre/, ou http:// www.conspiracywatch.info/11-Septembre-plus-d-
un-Francais-sur-quatre-croirait-a-1-implication-dugouvernement-americain a 1658.html, consultés le 13 février 2017. 


\title{
$R \cdot E ́ \cdot S \cdot U \cdot M \cdot E ́$
}

Militantisme en ligne, « complotisme » et disqualification médiatique : le cas de l'association ReOpen911 Cet article propose une approche interactionniste du conspirationnisme centrée sur les modalités concrètes de création et d'attribution du stigmate « complotiste ». Il s'appuie sur une enquête sur l'association ReOpen911. On y montre comment la forme ouverte de l'organisation (dans un espace de contestation autour des attentats du 11 septembre 2001 marqué par la présence d'acteurs d'extrême droite et/ou antisémites), la relative inexpérience des militants et leur stratégie d'échange avec les journalistes ont contribué à la construction et l'entretien du stigmate.

\begin{abstract}
In Dubious Battle

Online activism, « conspiracism » and disqualification in the media: the French ReOpen911 organization case This paper offers an interactionist approach of conspiracism, focused on the modalities of creation and attribution of the "conspiracist" stigma. It is based on the case of the French ReOpen911 organization. We describe here how the organization's being open (in a field of contestation around the September 11 attacks marked by the presence of far right and/or antisemitic actors), the relative inexperience of the activists and their strategy of communication with journalists have contributed to the construction and maintenance of the stigma.
\end{abstract}


